\title{
Estimation method of unbalanced power grid voltage in PWM Rectifier control system
}

\author{
Wen-Shao $\mathrm{Bu}^{\dagger}$ and Pei-Pei Dong \\ College of Electrical Engineering, Henan University of Science \&Technology \\ Luoyang 471003, Henan, China \\ ${ }^{\dagger}$ E-mail: wsbu@163.com
}

\begin{abstract}
To achieve the self-sensing detection of unbalanced power grid voltage, control system under the unbalanced power grid conditions are analyzed. Then the positive and negative sequence virtual flux of power grid is separated; then an estimation algorithm of unbalanced power grid voltage is presented by the positive and negative sequence virtual flux components. In addition, the unbalanced power grid voltage sensorless control system is constructed. The simulation results show that the accurate self-sensing detection of unbalanced power grid voltage can be realized; and in the steady state, a high stability can be obtained in the DC side voltage of PWM rectifier.
\end{abstract}

Keywords: PWM rectifier; Unbalanced power grid voltage; Self-sensing detection; Positive and negative sequence separation

\section{Introduction}

The voltage type three-phase rectifier using PWM control technology, i.e. VSR has a serious of characteristics, such as input current being sine wave, operation in unity power factor, and bidirectional flow of power [1]. Then, some industrial fields, such as industrial frequency converter have put an urgent need for the PWM rectifier technology [2-3]. Three-phase power grid is often in unbalanced station [2]. To simplify the controller of VSR, the influence of unbalanced situation is rarely considered. For some large capacity converters, if the unbalanced conditions were ignored, the produced unbalanced three-phase currents may lead to the increase of system loss and performance drop. In order to overcome the influence of unbalanced power grid, the control strategies of VSR under unbalanced grid conditions have been studied and gratifying research progress has been made [2, 3]. In VSR control system, three kinds of sensors are needed; include AC electromotive force sensor or power grid voltage sensor, AC current sensor, DC voltage sensor. More sensors not only increase the system cost, but also reduce the system reliability. To achieve the unity power factor or variable power factor control of VSR, the power grid voltage sensor is mainly used to detect the electromotive force of three-phase power grid, and supply a synchronous signal source for VSR [4]. If the power grid voltage 
sensor can be omitted, the system cost can be effectively reduced. However, most of the existing researches on power grid voltage sensorless control technology have not considered the unbalanced problem of power grid [4-5].

In this paper, considering the conditions of unbalanced power grid, AC voltage sensorless control technology of VSR is studied; an unbalanced power grid voltage method is presented.

\subsection{Control strategy of VSR with non AC voltage sensor}

\subsection{Control of VSR under the unbalanced power grid conditions}

The unbalanced three-phase power grid voltage or power grid electromotive force (EMF) $\boldsymbol{E}$ can be decomposed into three parts, include positive electromotive force $E^{P}$, negative sequence electromotive force $E^{N}$ and zero sequence electromotive force $E^{0}$. I.e. $E=E^{P}+E^{N}+E^{0}$, the corresponding time domain expression is as follow:

$$
\left[\begin{array}{l}
e_{a} \\
e_{b} \\
e_{c}
\end{array}\right]=E_{m}^{P}\left[\begin{array}{l}
\cos \left(\omega t+\alpha_{p}\right) \\
\cos \left(\omega t+\alpha_{p}-120^{\circ}\right) \\
\cos \left(\omega t+\alpha_{p}+120^{\circ}\right)
\end{array}\right]+E_{m}^{N}\left[\begin{array}{l}
\cos \left(\omega t+\alpha_{N}\right) \\
\cos \left(\omega t+\alpha_{N}+120^{\circ}\right) \\
\cos \left(\omega t+\alpha_{N}-120^{\circ}\right)
\end{array}\right]+E_{m}^{0}\left[\begin{array}{l}
\cos \left(\omega t+\alpha_{0}\right) \\
\cos \left(\omega t+\alpha_{0}\right) \\
\cos \left(\omega t+\alpha_{0}\right)
\end{array}\right]
$$

In (1): $E_{m}^{p}, E_{m}^{N}$ and $E_{m}^{0}$ are the respective peak values of positive, negative and zero sequence EMF; $a_{p}, a_{N}$ and $a_{0}$ are the respective initial phase angles of positive sequence, negative sequence and zero sequence EMF.

Because there is no channel for zero sequence current, the effect of zero sequence EMF is not considered. To achieve the control of VSR under unbalanced conditions, a key step is to separate the positive and negative sequence EMF of power grid. In this paper, a delay method is used to separate the positive and negative sequence EMF, the algorithms are as follows:

$$
\begin{aligned}
e_{a}^{p}(t) & =\left[e_{a}(t)+e_{b}(t-2 T / 3)+e_{c}(t-T / 3)\right] / 3 \\
e_{b}^{p}(t) & =\left[e_{a}(t-T / 3)+e_{b}(t)+e_{c}(t-2 T / 3)\right] / 3 \\
e_{c}^{p}(t) & =\left[e_{a}(t-2 T / 3)+e_{b}(t-T / 3)+e_{c}(t)\right] / 3 \\
e_{a}^{N}(t) & =\left[e_{a}(t)+e_{b}(t-T / 3)+e_{c}(t-2 T / 3)\right] / 3 \\
e_{b}^{N}(t) & =\left[e_{a}(t-2 T / 3)+e_{b}(t)+e_{c}(t-T / 3)\right] / 3 \\
e_{c}^{N}(t) & =\left[e_{a}(t-T / 3)+e_{b}(t-2 T / 3)+e_{c}(t)\right] / 3
\end{aligned}
$$

Where: $T$ is the set delay time constant.

To simplify the design of control system, by coordinate transformation, the three-phase coordinate system $(a, b, c)$ is converted into the $(d, q)$ coordinate 
system which is based on the fundamental frequency of the power grid. The specific transformation algorithm is as follow:

$$
\left[\begin{array}{l}
e_{a} \\
e_{b} \\
e_{c}
\end{array}\right]=C_{23} R(-\theta)\left[\begin{array}{l}
e_{d}^{p} \\
e_{q}^{p}
\end{array}\right]+C_{23} R(\theta)\left[\begin{array}{l}
e_{d}^{N} \\
e_{q}^{N}
\end{array}\right]
$$

Where: $C_{23}$ is the coordinate transformation matrix from two-phase to threephase; $R(\theta)$ and $R(-\theta)$ are the forward and reverse rotating coordinate transformation matrix; $e_{d}^{p} 、 e_{q}^{p}$ are the components of positive sequence fundamental EMF; $e_{d}^{N} 、 e_{q}^{N}$ are the components of negative sequence EMF.

In $\alpha \beta$ complex plane, complex vector $E_{\alpha \beta}$ can be expressed as:

$$
E_{\alpha \beta}=2\left[e_{a}+e_{b} e^{j 2 \pi / 3}+e_{c} e^{-j 2 \pi / 3}\right] / 3
$$

Equation (3) can be rewritten as follow:

$$
E_{\alpha \beta}=e^{j \omega t} E_{d q}^{p}+e^{-j \omega t} E_{d q}^{N}
$$

Where: $\omega$ is angular frequency; $E_{d q}^{p} 、 E_{d q}^{N}$ are the complex vectors of positive and negative sequence EMF. Expressions of $E_{d q}^{p}$ and $E_{d q}^{N}$ are as follows:

$$
E_{d q}^{p}=e_{d}^{p}+j e_{q}^{p}, \quad E_{d q}^{N}=e_{d}^{N}+j e_{q}^{N}
$$

According to the topology of VSR, the complex vector of power grid EMF in $\alpha \beta$ coordinate system can be expressed as follows:

$$
\begin{gathered}
E_{\alpha \beta}=V_{\alpha \beta}+L\left(d I_{\alpha \beta} / d t\right)+R I_{\alpha \beta} \\
\left\{\begin{array}{l}
V_{\alpha \beta}=2\left[v_{a}+v_{b} e^{j 2 \pi / 3}+v_{c} e^{-j 2 \pi / 3}\right] / 3 \\
I_{\alpha \beta}=2\left[i_{a}+i_{b} e^{j 2 \pi / 3}+i_{c} e^{-j 2 \pi / 3}\right] / 3
\end{array}\right.
\end{gathered}
$$

Where: $V_{\alpha \beta}$ and $I_{\alpha \beta}$ are the $\mathrm{AC}$ voltage complex vector and $\mathrm{AC}$ current complex vector of VSR. Equation (7) can be rewritten with the vector in $d q$ as follow:

$$
\left\{\begin{array}{l}
V_{\alpha \beta}=V_{d q}^{P} e^{j \omega t}+V_{d q}^{N} e^{-j \omega t} \\
I_{\alpha \beta}=I_{d q}^{P} e^{j \omega t}+I_{d q}^{N} e^{-j \omega t}
\end{array}\right.
$$

Where: $V_{d q}^{p}$ and $V_{d q}^{N}$ are the complex vectors of positive and negative sequence AC side voltage. $V_{d q}^{p}$ and $V_{d q}^{N}$ can be expressed as follows: 


$$
V_{d q}^{p}=v_{d}^{p}+j v_{q}^{p}, \quad V_{d q}^{N}=v_{d}^{N}+j v_{q}^{N}, \quad I_{d q}^{p}=i_{d}^{p}+j i_{q}^{p}, \quad I_{d q}^{N}=i_{d}^{N}+j i_{q}^{N}
$$

According to (4), (6) and (7), there are following equations:

$$
\left\{\begin{array}{l}
E_{d q}^{p}=L d I_{d q}^{p} / d t+R I_{d q}^{p}+j \omega L I_{d q}^{p}+V_{d q}^{p} \\
E_{d q}^{N}=L d I_{d q}^{N} / d t+R I_{d q}^{N}+j \omega L I_{d q}^{N}+V_{d q}^{N}
\end{array}\right.
$$

When the power grid is unbalanced, the active power and reactive power on the power grid side of VSR can be expressed as follows:

$$
\left\{\begin{array}{l}
P_{0}=1.5\left(e_{d}^{p} i_{d}^{p}+e_{q}^{p} i_{q}^{p}+e_{d}^{N} i_{d}^{N}+e_{q}^{N} i_{q}^{N}\right) \\
P_{c 2}=1.5\left(e_{d}^{p} i_{d}^{N}+e_{q}^{p} i_{q}^{N}+e_{d}^{N} i_{d}^{p}+e_{q}^{N} i_{q}^{p}\right) \\
P_{s 2}=1.5\left(e_{q}^{N} i_{d}^{p}-e_{d}^{N} i_{q}^{p}-e_{q}^{p} i_{d}^{N}+e_{d}^{p} i_{q}^{N}\right) \\
q_{0}=1.5\left(e_{q}^{p} i_{d}^{p}-e_{d}^{p} i_{q}^{p}+e_{q}^{N} i_{d}^{N}-e_{d}^{N} i_{q}^{N}\right) \\
q_{c 2}=1.5\left(e_{q}^{p} i_{d}^{N}-e_{d}^{p} i_{q}^{N}+e_{q}^{N} i_{d}^{p}-e_{d}^{N} i_{q}^{p}\right) \\
q_{s 2}=1.5\left(e_{d}^{p} i_{d}^{N}+e_{q}^{p} i_{q}^{N}-e_{d}^{N} i_{d}^{p}-e_{q}^{N} i_{q}^{p}\right)
\end{array}\right.
$$

Where: $p_{0}$ and $q_{0}$ are the averages of active and reactive powers. $p_{c 2}$ and $p_{s 2}$ are the cosine term harmonic peak and the since term harmonic peak of second order active power; $q_{c 2}$ and $q_{s 2}$ are the cosine term harmonic peak and the since term harmonic peak of second order reactive power.

When the power grid is unbalanced, there exist second order harmonic components in the DC side voltage. When the control performance requirement of DC voltage is high, $p_{s 2}$ and $p_{c 2}$ should be suppressed. Taking into account the active and reactive power instructions $p_{0}^{*}=q_{0}^{*}=0, p_{s 2}^{*}=0$ and $p_{c 2}^{*}=0$, the relevant current instructions, such as $i_{d}^{p^{*}}, i_{q}^{p^{*}}, i_{d}^{N^{*}}$ and $i_{q}^{N^{*}}$, can be derived:

$$
\left[\begin{array}{l}
i_{d}^{p^{*}} \\
i_{q}^{p^{*}} \\
i_{d}^{N^{*}} \\
i_{q}^{N^{*}}
\end{array}\right]=\left[\begin{array}{cccc}
e_{d}^{p} & e_{q}^{p} & e_{d}^{N} & e_{q}^{N} \\
e_{q}^{p} & -e_{d}^{p} & e_{q}^{N} & -e_{d}^{N} \\
e_{q}^{N} & -e_{d}^{N} & -e_{q}^{p} & e_{d}^{p} \\
e_{d}^{N} & e_{q}^{N} & e_{d}^{p} & e_{q}^{p}
\end{array}\right]\left[\begin{array}{l}
\frac{2}{3} p_{0}^{*} \\
0 \\
0 \\
0
\end{array}\right]=\frac{2 p_{0}^{*}}{3 D}\left[\begin{array}{l}
e_{d}^{p} \\
e_{q}^{p} \\
-e_{d}^{N} \\
-e_{q}^{N}
\end{array}\right]
$$

Where: $D=\left[\left(e_{d}^{p}\right)^{2}+\left(e_{q}^{p}\right)^{2}\right]-\left[\left(e_{d}^{N}\right)^{2}+\left(e_{q}^{N}\right)^{2}\right] \neq 0$.

If PI regulator is used for current inner loop, according to feed forward decoupling law, the control instructions of positive sequence and negative sequence voltages on AC side can be derived: 


$$
\left\{\begin{array}{l}
v_{q}^{p^{*}}=-\left(K_{i p}+K_{i I} / s\right)\left(i_{q}^{p^{*}}-i_{q}^{p}\right)-\omega L I_{d}^{p}+e_{q}^{p} \\
v_{d}^{p^{*}}=-\left(K_{i p}+K_{i I} / s\right)\left(i_{d}^{p^{*}}-i_{d}^{p}\right)+\omega L I_{q}^{p}+e_{d}^{p} \\
v_{d}^{N^{*}}=e_{d}^{N}-\left(K_{i p}+K_{i I} / s\right)\left(i_{d}^{N^{*}}-i_{d}^{N}\right)-\omega L i_{q}^{N} \\
v_{q}^{N^{*}}=e_{q}^{N}-\left(K_{i p}+K_{i I} / s\right)\left(i_{q}^{N^{*}}-i_{q}^{N}\right)+\omega L i_{d}^{N}
\end{array}\right.
$$

Similar with the power grid voltage, the positive and negative sequence currents should be detected separately also.

\subsection{Self-sensing detection of AC voltage}

The power grid side of VSR can be seen as a virtual electric machine, from the virtual flux-linkage, the power grid voltage can be observed. Ignoring the AC side resistance, from the relationship between virtual flux-linkage and induced electromotive force, there are following equations:

$$
\varphi_{a}=L i_{a}+\int u_{a} d t, \quad \varphi_{b}=L i_{b}+\int u_{b} d t, \quad \varphi_{b}=L i_{b}+\int u_{b} d t
$$

Where: $i_{a}, i_{b}$ and $i_{c}$ are three phase currents; $u_{a}, u_{b}$ and $u_{c}$ are the AC side voltages of VSR.

The AC side voltages $u_{a}, u_{b}$ and $u_{c}$ can be expressed with $U_{\mathrm{dc}}$ and switching functions $s_{a}, s_{b}$ and $s_{c}$, corresponding expressions are as follows:

$$
u_{a}=U_{d c} s_{a}, \quad u_{b}=U_{d c} s_{b}, \quad u_{c}=U_{d c} s_{c}
$$

Putting (15) into (14), then:

$$
\varphi_{a}=L i_{a}+\int U_{d c} s_{a} d t, \quad \varphi_{b}=L i_{b}+\int U_{d c} s_{b} d t, \quad \varphi_{c}=L i_{c}+\int U_{d c} s_{c} d t
$$




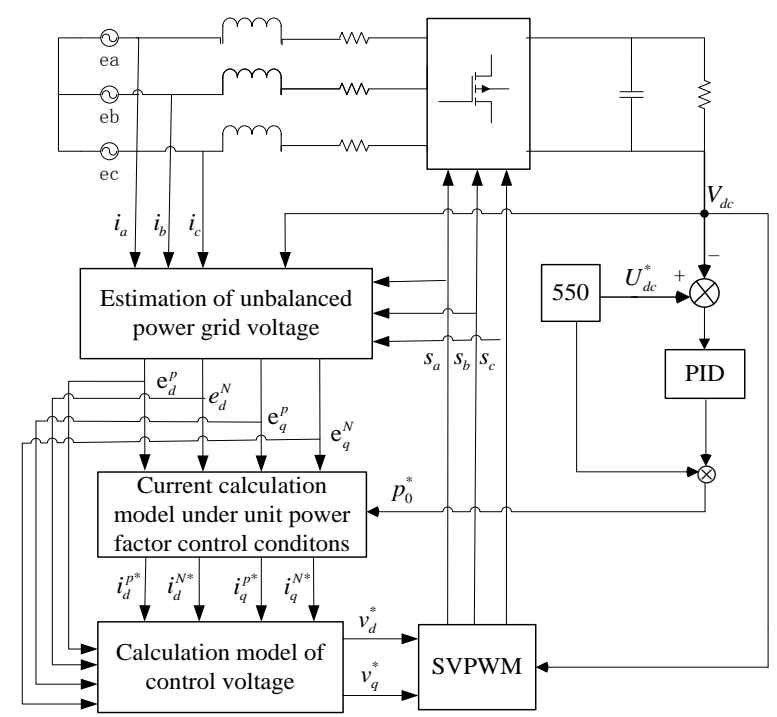

Fig.1 Power grid voltage sensorless control system under unbalanced power grid condition

According to (16), virtual flux-linkage $\varphi_{a}, \varphi_{b}$ and $\varphi_{c}$ can be calculated. But the switching functions $s_{a}, s_{b}$ and $s_{c}$ are PWM signal with high frequency, signal process is complicated. So in this paper, the switching functions are replaced with duty ratio $d_{a}, d_{b}$ and $d_{c}$. Then (16) can be rewritten as follows:

$$
\varphi_{a}=L i_{a}+\int U_{d c} d d t, \quad \varphi_{b}=L i_{b}+\int U_{d c} d_{b} d t, \quad \varphi_{c}=L i_{c}+\int U_{d c} d d t
$$

The delay method is effective for the positive and negative sequence separation of power grid virtual flux, and the $\varphi_{a} \varphi_{b}$ and $\varphi_{c}$ are separated into $\varphi_{a}^{+}, \varphi_{a}^{-}, \varphi_{b}^{+}, \varphi_{b}^{-}, \varphi_{c}^{+}$, and $\varphi_{c}^{-}$. By $3 / 2$, positive and negative sequence rotating transformation, $\varphi_{a}^{+}, \varphi_{a}^{-}, \varphi_{b}^{+}, \varphi_{b}^{-}, \varphi_{c}^{+}$, and $\varphi_{c}^{-}$are transformed to $\varphi_{d}^{+}, \varphi_{q}^{+}, \varphi_{d}^{-}$and $\varphi_{q}^{-}$. From the obtained virtual flux, the voltage on power grid side can be obtained:

$$
\begin{gathered}
\varphi_{\alpha \beta}=\varphi_{d q}^{p} e^{j \omega t}+\varphi_{d q}^{N} e^{-j \omega t} \\
\varphi_{d q}^{p}=\varphi_{d}^{p}+j \varphi_{q}^{p}, \quad \varphi_{d q}^{N}=\varphi_{d}^{N}+j \varphi_{q}^{N} \\
E_{\alpha \beta}=d \varphi_{\alpha \beta} / d t
\end{gathered}
$$

Substituting (18)-(19) into (20), then:

$$
E_{\alpha \beta}=j \omega \varphi_{d q}^{p} e^{j \omega t}-j \omega \varphi_{d q}^{N} e^{-j \omega t}
$$

Substituting (19) into (21) and then substituting (21) into (20), then: 


$$
E_{d}^{p}=-\omega \varphi_{q}^{p}, \quad E_{q}^{p}=\omega \varphi_{d}^{p}, \quad E_{d}^{N}=\omega \varphi_{q}^{N}, \quad E_{q}^{N}=-\omega \varphi_{d}^{N}
$$

From (22), the power grid AC voltage can be estimated. Then AC voltage sensorless control can be realized under the unbalanced power grid conditions. Fig. 1 shows the principle diagram of system.

\section{Simulation Analysis}

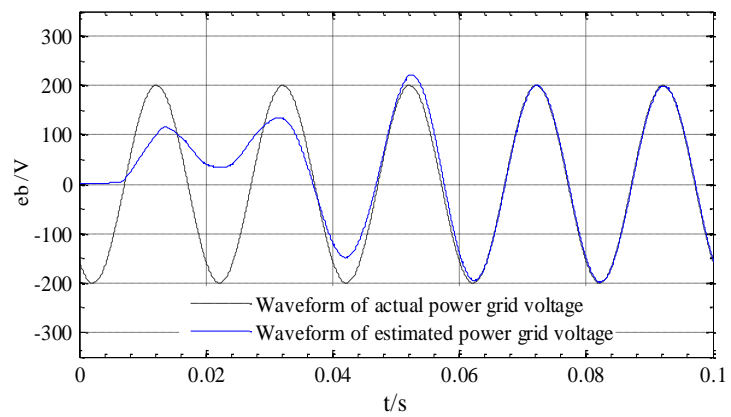

Fig. 2 comparison of $e_{\mathrm{b}}$ voltage between measured valued and observed value

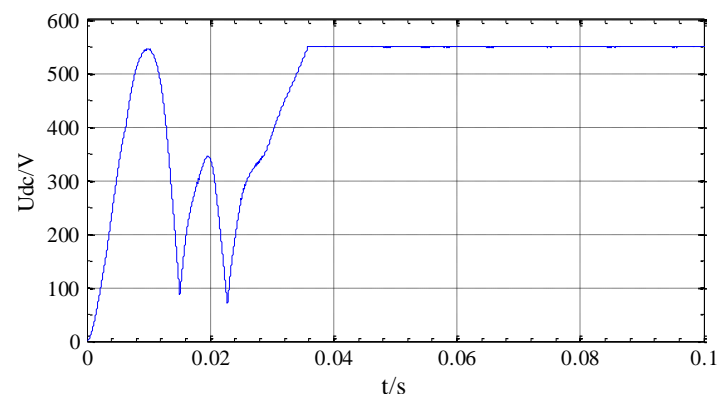

Fig.3 DC voltage waveform of power grid voltage sensorless control system

According to Fig.1, the simulation analysis is made. The main parameters are set as follows: frequency of Power grid 50HZ. Unbalanced phase voltages $e_{a}=220 \cos (\omega t), \quad e_{b}=200 \cos (\omega t-129.1), \quad e_{c}=213.5 \cos (\omega t+114)$; given DC voltage 550V. From the simulation results in Fig.2 and Fig.3, it can be seen that in addition to the initial stage of voltage estimation, the restructured power grid voltage is fully accordant with the actual voltage either in amplitude or in phase. During the start-up stage, there is a transient DC voltage fluctuation. In the steady state, the DC voltage is always being kept about the set value.

\section{Conclusion}

The control method of VSR under unbalanced conditions is analyzed firstly. Then by delay method, the positive and negative sequence separation of virtual 
flux linkage is carried out. Using positive and negative sequence virtual fluxlinkage, the unbalanced power grid voltage is estimated. From the system simulation results, it is known that: after a short adjustment process in the starting stage, fast self-sensing detection of unbalanced grid voltage can be achieved; In steady state, the estimated power grid voltage is accordant with the actual power grid either in amplitude or in phase; the presented sensorless control strategy not only is effective.

In addition, there exists a fluctuation in DC voltage during the starting stage. This is due to the voltage estimation need an adjustment time.

\section{Acknowledgement}

The supports of International Cooperation Project on Sci. and Tech. of Henan Province (114300510029), and Nature Science Fund of Henan Province Education Bureau (2010B510011), are acknowledged.

\section{References}

[1] P.S. Das, K.H Kim. Real-time multiple open-switch fault diagnosis in threephase AC/DC PWM converter for PMSG based grid-connected wind power generation system, Int. J. Control Autom., 2014, Vol.7, No.9, pp. 329-344.

[2] H.J ZHANG, J ZHANG, Z.M SUN. Study of control strategy based threephase PWM rectifier under unbalanced input voltage condition. Power system protection and control, 2009, Vol.37, No.22, pp.13-16.

[3] Marcos B. Ketzer, Cursino B. Jacobina. Sensorless Control Technique for PWM Rectifiers with Voltage Disturbance Rejection and Adaptive Power Factor, IEEE Trans on Industrial Electronics, 2015, Vol.62, No.2, pp.1140-1151.

[4] W.S BU, L.L ZHAI. Research on Power Grid Voltage Sensorless DPC of PWM Rectifier, Electric drive, 2013, Vol.43, No.2, pp.56-60.

[5] J.H WANG, X.Y YANG. Control Strategy of Voltage Source PWM Rectifiers under Unbalanced Voltage Conditions, Proceedings of the CSEE, 2011, Vol.31, No.18, pp.14-20. 